\title{
$\mathfrak{n} \mathfrak{h a l t}$.
}

\section{BHegerlibes Redt.}

\section{a. IReiduredt.}

Nx.

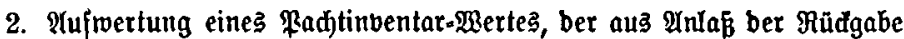
ber Radjtung in ßapiermarl gejdäßst murbe. Periłdfidjtigung bes 11 mitanbs, baß ber ฬähter ben ßadjzins in entwertetem Selbe gezahlt hat? .......................

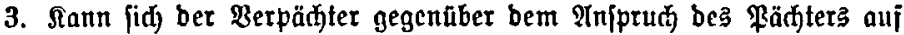

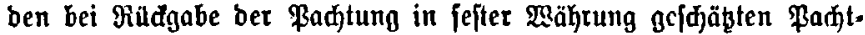
inbentar-Preiş barauf berujen, baß̧ ber Päđter ben Pađtzing in ent. wertetem Gelbe gezahlt habe? ................ 10

4. Jntwieweit hinbert bie Cintragung ber Bormerfung einer Şypolbef,

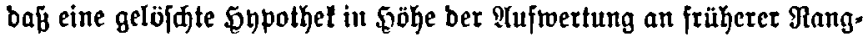
ftclle wieber eingetragen witb?...............

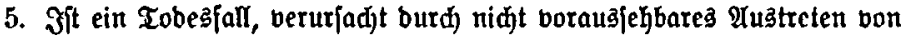
T̃ohlenochbgas aus einem Bimmerofen, als llnjall im Finne ber all.

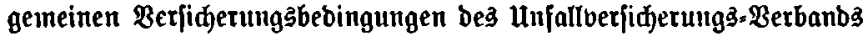
anzufehen? Bergiftung als Unfall? . . . . . . . . . . .

6. Unter twelden Borauşeşungen gilt für bie ßarteien eines Mietberhält= niffes bie gelebliche Miete?.................

7. Über ben Ginflußs ber unheilbaren Niatigteit bon Generalberfamm=

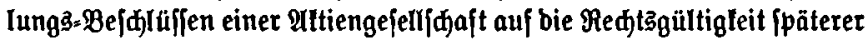
berartiger $\mathfrak{g e f d j l u ̈ f f e ~ . ~ . ~ . ~ . ~ . ~ . ~ . ~ . ~ . ~ . ~ . ~ . ~ . ~ . ~ . ~ . ~}$

8. Begeht ein in soppelehe lebenber (5hemann buta gejaledtlidgen Ber. leht mit feiner nunmehrigen Frau fajon bann einen bie Sheibung ber früheren (5ुhe redtfertigenben (5hebruक, wenn jeine Innahme, bieje Ehe fei niðtig, auf fahrläfligleit beruht, ober liegt ein foldjer çhe. 
9x.

brud, nur bann bor, wenu er bie Fedtsbeftänbigleit feiner früheren Ehe Iennt?...................... 35

9. Seht ein ๔đaben, ber baburd) berurjadyt worben ift, baß̧ bas હdiff

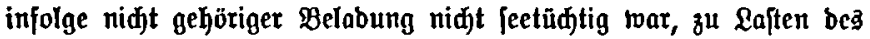
Berfiderers ober bes Berfiderunganehmers? . . . . . . . .

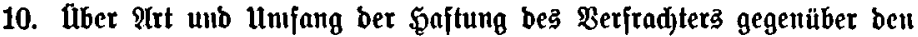

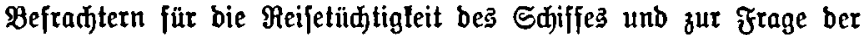
Freizeidnung bes Berfradters bon biefer $\mathfrak{E a f t u n g}$. . . . . . . .

11. luber bie Unterlaf\{ungłlage, bie ein Berbanb zur Förberung getocrb. liđer Эntereffen (\$13 uniws.) anftrengt. Cteht ihr ber Umftanb ent. gegen, baß gegen benfelben Beflagten (d)on ber burd beffen 2 Bettbetoerb unmittelbar Bcrlebte Slage auf Unterlaffung erhoben hat?

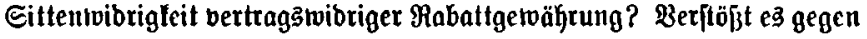
bie guten Sitten, wenn bem bertragšuntreuen ânehmer (Mieberverfäufer) für ben Fall ber 9tidteinhaltung ber "Martenpreife" Liefer-

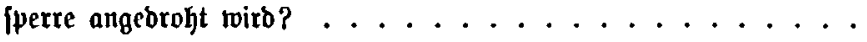

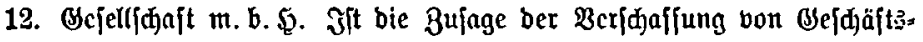

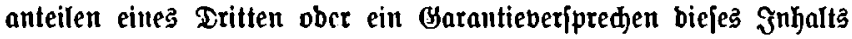
formpflidtig nad) $\$ 15$ glbf. 4 ßmbsç . . . . . . . . .

15. $\mathfrak{B a} a \mathfrak{s}$ muß ein Beamter z" friner Crntlaftung beweifen, wemu er fiit bas 9tbhanbenlommen einet in [eine amtlidje Obhut gelangten Eadje verantwortlid gemadt miro ?. . . . . . . . . . . .

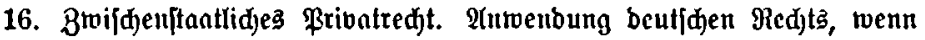

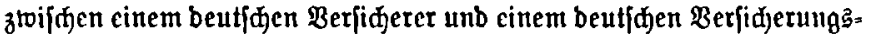

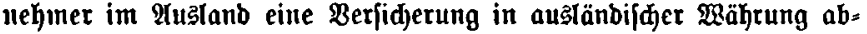
gefdjloffen worben ift? thernabme ber in Dfterreid abgefdloffenten Ber-

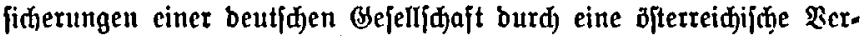
fidjcrungägefellfdaft; Befreiung ber beutjden Befell[daft bon ber

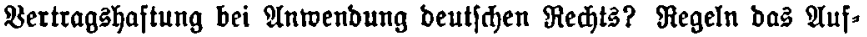
wertungägefeß und bie bazu erlaffenen Berotbnungen aud) bie श(uf.

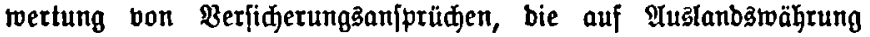

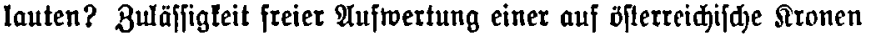
Iautenben frotberung nad beutfacm Redt? . . . . . . .

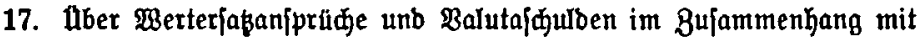
bem Berfall ber beutjden währung ...........

19. Rann ein im Эahte 1917 bon einer preußjifłen Stabtgemeinbe für Sriegszzwede aufgenommenes Darleken, wofür ber Darlehensgeber als Sidjerheit an Stelle eines Sdjulbfacinz ein Sparlaffenbud ber 
$9 x$.

ftäbtifden Sparfafie erfalten hat, alł ভduulbjdein: Darlehen im Einute

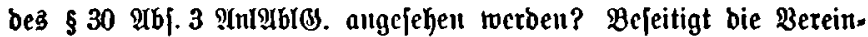

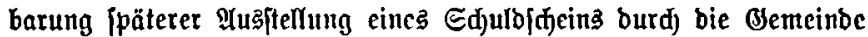
ben Mangel eines folden? Begriff bes Sparguthabens im Sinne bes

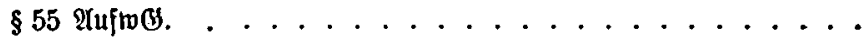

21. Gind $\$ 1$ Uu॥

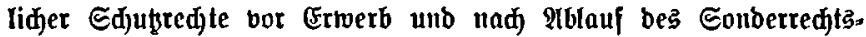

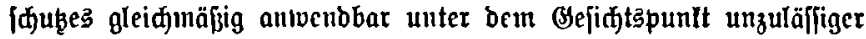

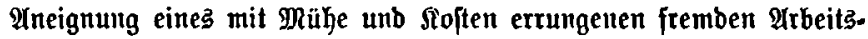

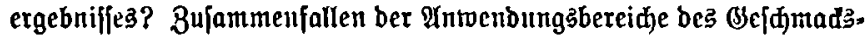
muftergejeges unb bes unlmss.? Mann ftellt bie Radbillbung eines

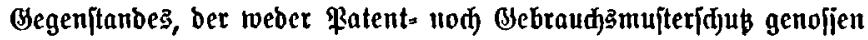

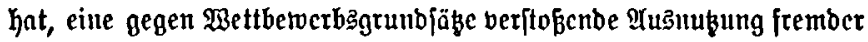

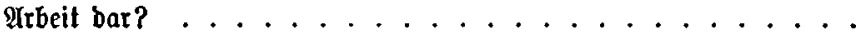

22. Llber Tarifberedunng bei Chemifalien, wenn zwar ber berjanbte ভtofi eine anbere demifde Bcrbindung ift als ber im Tarif gennnte, weln aber beibe nus bem un̈ulidjen abjallerzengnis hergcitellt und in gleidjer \$Beife mirtidjaftlid) berwertet werben . . . . . . . 101

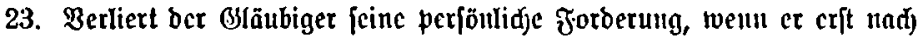
bem 1. Jantuar 1926 bell perjönlidjen Edjulbner ber Thujtocrtungs.

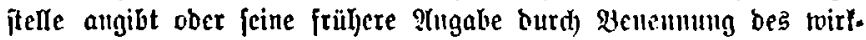
lidgen Edulbners beridtigt? . . . . . . . . . . .

24. Wirft bie Bernutung bes $\$ 1362$ 9Ib 1 25(SB). aud) bnut, wenll ber

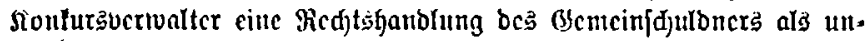

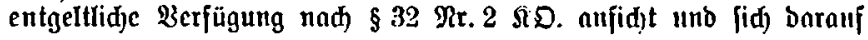
beruft, bafi bie bout ber Ġhejrau bem Mann alz angeblide Gegenleiftung gemährten Cadhen von bornhercin beffen Eigentum getvefen frien? .

25. Sìt bie Cintraguıg ber \$iänoung obcr ber Berpfänbung cincr bci

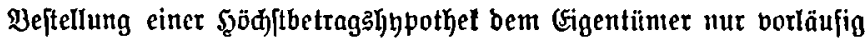
zuftehenben Cigentlintergrunbjdulb ohne mciteres beshalb unwirlfam, weil bie Cintragung wegen ber $\mathfrak{B o r j d}$ rift bes $\$ 40$ MBD. nidt hätte

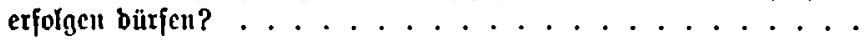

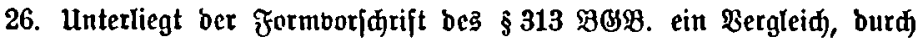
ben fid ber Sd)warzbertäufer nad) Yuflaffung unb Eintragung bes Säufers im Brumbbuđ verpflidtet, bas Brunbftüd bem গäufer zu belaffen und zur Crteilung ber nad bem preußifden Brunbitüđ马̈: verfehrs:-Befę erjorberliden behörbliden Benehmigung mitzumirlen?

27. Sann bas Brunbbuđamt burd, einftweilige Berfügung zur Bornałme

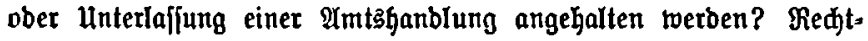


Mr.

liđhe Bebeutung einer einftweiligen Berjügung, bie bem fäufer eines Grunbftüđ马 berbietet, bie zum Gigentumserwerb erforberlide Gintragung im Grunbbud herbeizulühren. Intwenbbarleit bes $\$ 878$ B(3). auf ein burđ cinftreilige Berfügung begründetes Ertwerb3.

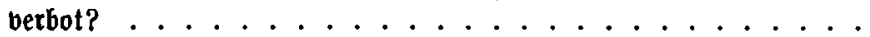

28. Ulber bie außerbertraglide Sorgfaltapflidt bez ভđjeppjijifahrtz.

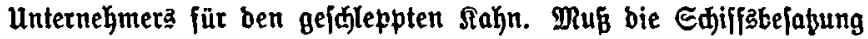
aud beim Fehlen einer vertragliden Beziehung zwijhen bem unter. neghmer unb bem Sakhneigner Gilfe leiften, wenn ber Sahn auf Grfudjen bes Sahnfdiffers an Land gejegt wirb unb zu finfen broht?

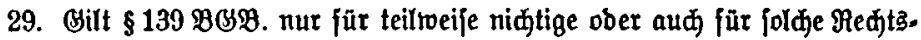
geidäfte, bie wegen Fehlens ber erforberliden Benehmigung teiltoeife unwirffam finb? Fam ber Bertragşgegner, ber gemäß $\$ 179$

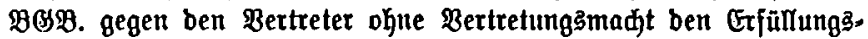

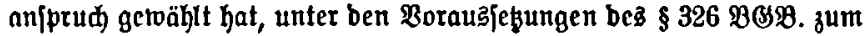
Sdjabenser|akan\{prud abergehen? Sajtung wegen Berjaulbenz beim

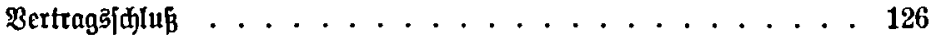

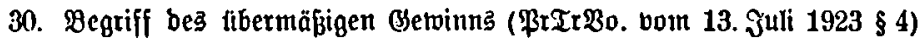
unb ber unangemeffenen Bergütung (Mieteridutg(G. \$49a) bei ber Bermietung von Räumen . . . . . . . . . .

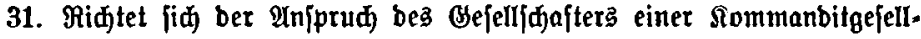

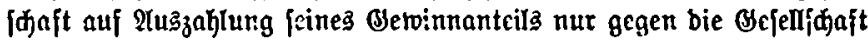
ober auch gegen bie anberen Befellfdafter? Finbet bie Borjarift beả

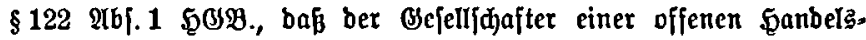

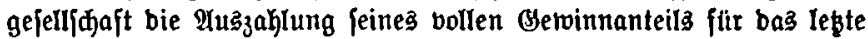

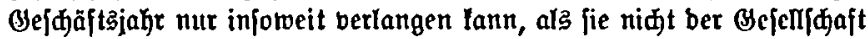
zum offenbaren Stjaben gereidt, auf ben Sommanbitiften 9lnwenbung,

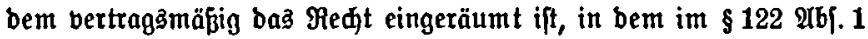
bezeidneten llmfang Belb aus ber Beferlidjafts̆affe zu erheben?. .

33. Liber bie Sittentwibrigleit eines Bertrags, worin ein gemeinmübiges

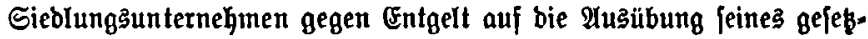
liden Borlaufżrchts verzidtet . . . . . . . . . . . .

34. Sant ber ß̈äubiger einer Shypothelenforberung bie im Saufpertrag über bas belajtete (S) runbitüđ vom (Frwerber erflärte હdulbubernabme nod) im Raufe bes afufwertungsberfahrens wirlfam genchmigen?

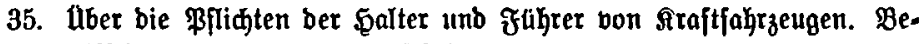

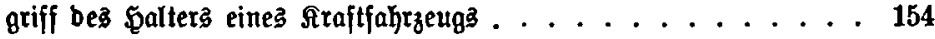

36. Wher bie Saftung einer Bemeinbe gegentiber einet Stanlenlaffe, bie ben Bremeinbeborjtanb mit ben Bsejđäften einer bon ihr eingeridteten 
Rr.

Bablftelle betraut hat, wenn ein von jenem in bet 8 afjlftelle befdadftigter Dritter bort eingegangene faffenbeiträge beruntreut . . . 162

37. Bebarf ein Saufbertrag über bas vom (Figentumer felbft zu fdilagenbe bolz eines \$albgrumbittids ber Benehmigung gemäß ber Betanntmađung über ben Berfehr mit lanbroirtjđaftliden Brrunbftüđen vom 15. Mär 1918? . . . . . . . . . . . . . . . .

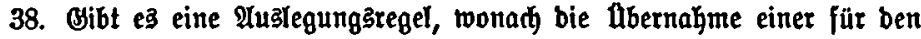
Berfäufer eingetragenen Eigentümergrunbjđulb in Antednung auf ber Saufpreis bie Saufpreisforberung nidt tilgt, fonbern nur lidjert?

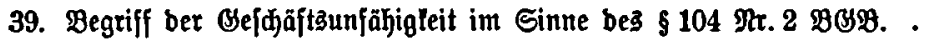

41. aber bie Bunttigleit von Sałungabeftimmungen einer Aft.-Bref., bie ben allmäbliden lubergang ber altienredte auf ben am unterneb̆men beteiligten Etaat bezweden. . . . . . . . . . . . 17

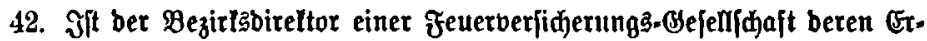

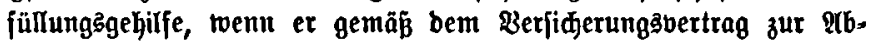
wenbung ober Pinberung bes Shabens Meifungen unb Berbote er.

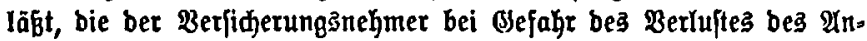

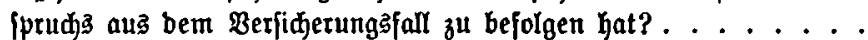

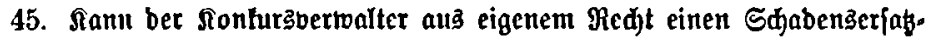
anjprud regen unerlaubter ఏanblung geltend maßjen, bie eine Eđhäbigung ber Gefamtheit ber STonfur gläubiger Kerbeigefühtt hat?

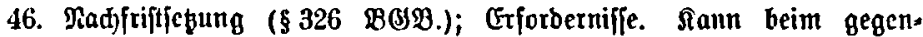
feitigen \$Bettrag, befonbera beim Sutzeffiblieferungägejhäft, ber im Berzug befinblidie Teil, wenn infwifhen eine Seiftung bez albern Teils fällig geworben ift, baraufbin bie eigene Leiftung bertweigern ober ein Buradbejaltungstedt geltenb madjen? ..........

48. Mann fann jemanb, ber fid flir einen anbern, ohne in beffen Єđulb -miteinzutreten, nur twedjelmäpig berpflidtet hat, auz bem Єđulb. berbältnis besิ anbern eine Finrebe gegen ben Medjelaniprud ab.

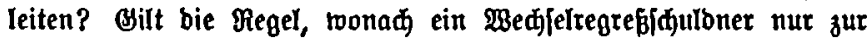
Tilgung feiner eigenen Berpflidtung zahlt, aud bann, wenn ber Bablenbe ber 2lusfteller bea Medjels ift unb ber Bezogene aus Befälligteit gegen ihn alzeptiert hat? Bum Begriff bez̉ Pfanbinbolfa= ments .................. 205

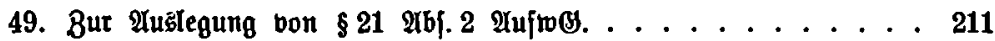

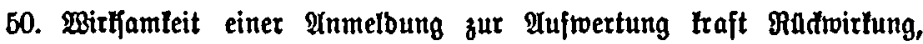

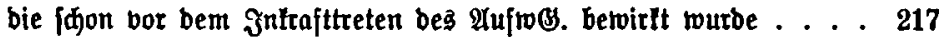


Mr.

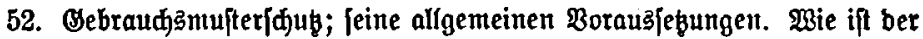

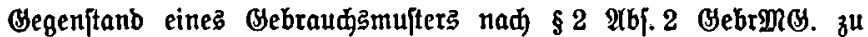
beftimmen? Ređttiđje Beurteilung von Sđuł̧ariprüळen, bie nad) Gintragung eines Bebraudjmu[tersె eingereidt worben finb ....

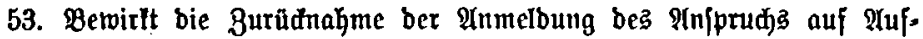

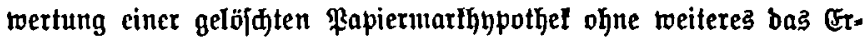

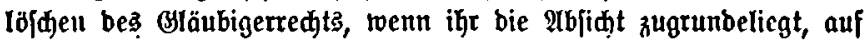
bie aufzumertenbe sghpothel zu verzidten, ober wirb ber Berzidit auf bie Iufwertungshgpothel erft twirfant, wenn er ins (srunobud)

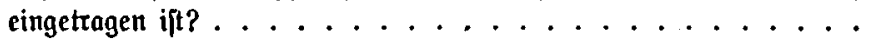

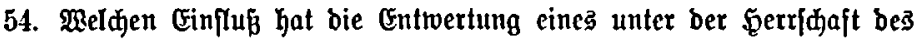
ßreußifden allgemeinen \&anbređts einer Sirdjengemeinbe bermadten Siapitals auf bie Fortbauer ber Berpflidturig ber Bemeinbe, ent: jpredgenb ber ihr vont Erblaffer gemadjten Auflage fein Erbbegräbuiz

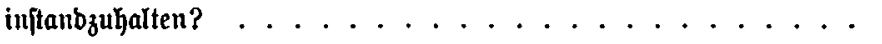

56. liber bic perfönlid)e Şaftung bcs iul eigenen Ramen berhanbelnben

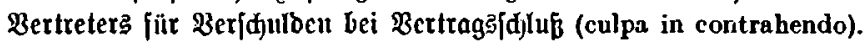

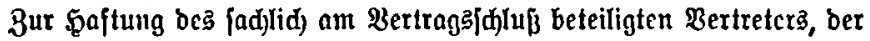
nus formalen olrimben für eincn anbert auftrift unb abjdyliept . .

57. Sit eine formlofe Bercinbarung über bie $9(u$ fwertung twirffam, und)=

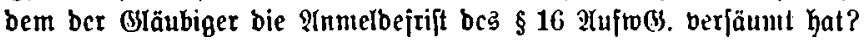

58. Ulber ఏnftuıg für Єđjabcn, bcr burd) eine verlehrsagcjährbenbe 2(nlage vcrurfant worben ift. Prima facie $=8$ 8eweis ...... 258

59. Samu ber ßatentingaber auf Unterlaffung flagen, weın ber Cingriff

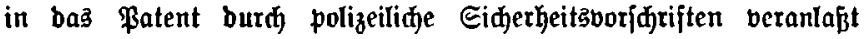

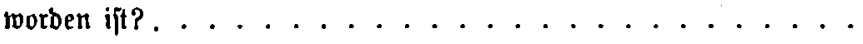

60. Uuflarhciten in Beförberungatarifen. Juternationales lubereinfommen tiber ben (Eifenbahn-gradjtberfehr. Begriff ber lumftationierung von

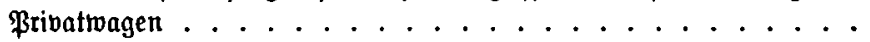

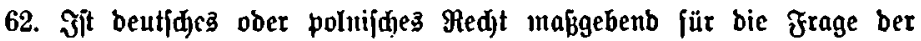
Ifufwertbarleit ber einem Jnlänber zuftehenben perjönliduen forbe: rung aus einem Marloarlehen, fuir bas int fpüter polnifd gewordenen Teil Ober[d]efiens fypothel beftellt worben war? . . . . . . .

63. Inu ber Sdjulbner, ber aus unfenntnis einer ihgm gegen ben Bräu. biger zuftchenben Forberung bie Tufredgnung berfäumt unb feine Sđuld an ben Bräubiger bezahlt hat, bas Bezahlte zurüfforbern unb nođ̆ nađ̆träglid aufrednen? . . . . . . . . . . 
Rr.

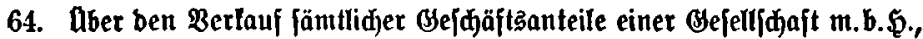

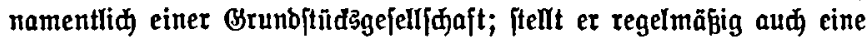
Beräukerung bes Unternekmens felbłt bar unb fimben babei aud ohne befonbere Bertragsbeftimmung bie gefestlidjen Borfdriften über

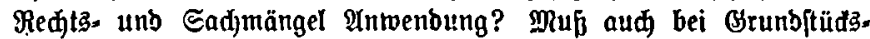

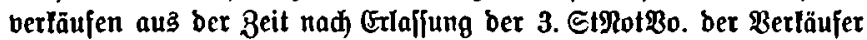

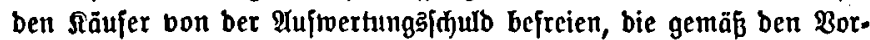

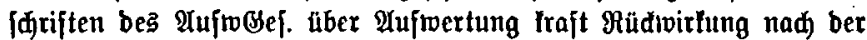
\&ōfकung bon Shbothelen beftehen geblieben ift? thber bie entipredenbe

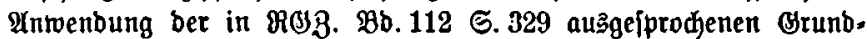

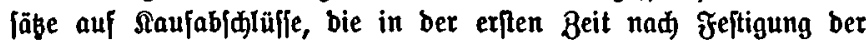
beutfden Bährung gegen einen toertbeftänbigen \$reis vorgenommen

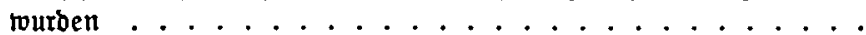

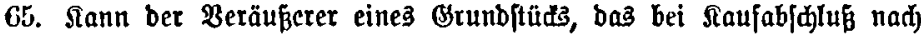

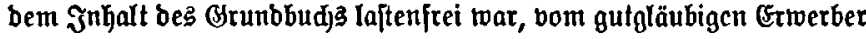

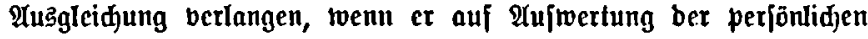

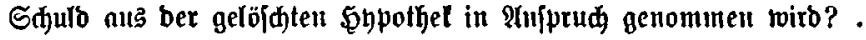

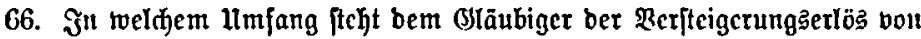
Mertpapieren zu, bie er vont feiner $\mathfrak{B a n l}$ zu verlangen hatte, lvenn bie Fapierc in Sonbon hinterlegt, bann toährend bes șriegcs verftcigert

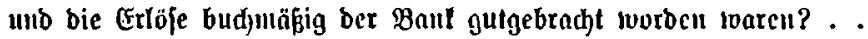

67. 3it cinc Sonfursjurbernng, bic cincm Mitglieb bes Porftanbs cincr

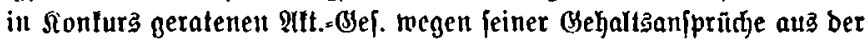

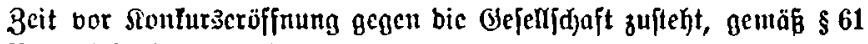
Mr. 1 ID. Vevorredtigt? . . . . . . . . . . . . .

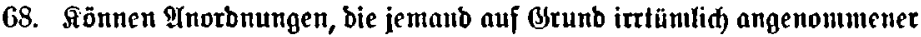

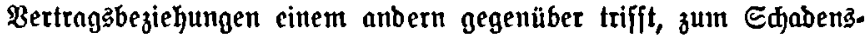
erfał aus unerlaubter (janblung berpflidten? Unterjajieb zwijd)en Zornahme eines Staat3hoheitzaltés unb einer Maßnahme ber ftaat. liden Bermögenzberwaltung. Offizier als verfaffungäntäßig be= rufener Bertreter bes \$reidjes? . . . . . . . . . . .

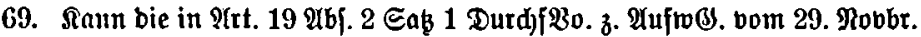
1925 vorgejehene Bereinbarung aud nod nad ber (Finzahlung auf bas Eparlafjenbud wirlfam getroffen werben? Sit fur bie $\mathfrak{A} u$;

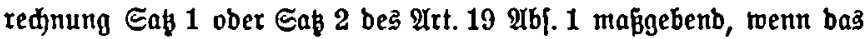
Eparlaffenguthaben zroar vor bem 15. Juni 1922 erworben, aber bie Bereinbarung, Daß es zur Abtragung einer shpothel nber Grunb|dulb 3u bienen beftimnt fei, erft nad) bem 14. Suni 1922 getroffen wurbe?

70. Eifenbahnfradtberlehr. Sïnnen tortbollere frelle als Soltbarfeiten gelten? $\mathfrak{M u k \beta}$ bie Bahn bei Trnnahme eines Fradtftuda, als beffen 
gx.

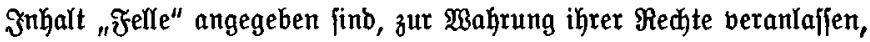

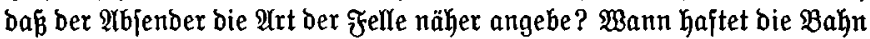

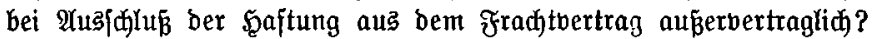
Ft bei gleidgzeitigem 2tbhandenfommen bon Fradtgut und Frarhtbrief bis auf weiteres anzunehmen, daß $\mathfrak{B a h n a n g e f t e l l t e ~ b a b e i ~ b e t e i l i g t ~ f i n d ? ~}$

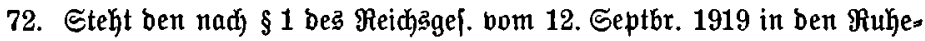
ftanb verjebten Beamten ein lebenslänglider $2(n$ pprud auf zehn=

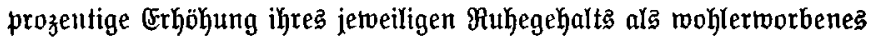

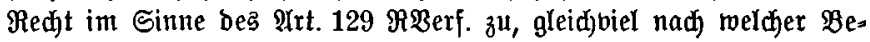
folbungsorbnung bas Ruhegehalt beredinet ift? . . . . . . .

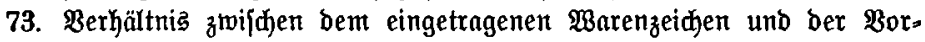
benukung bes geidens . . . . . . . . . . . .

74. Sit bei Beredhung bes Bolbmarfbetrags einer aufzuwertenben $\mathfrak{Q}$ ypo= thef ber Erwerb auf Brund ber Auseinanderfekung einer offenen

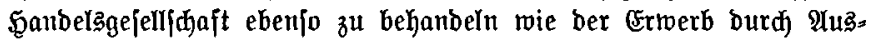
einanderfekung einer Bemeinjdaft im Sinne von $\$ 3$ abj. 1 Mr. 4 Áufwos.? . . . . . . . . . . . . . .

75. Begrünbet bie burdh den Liquibator einer Sejellydaft m. b. מ̨. bes

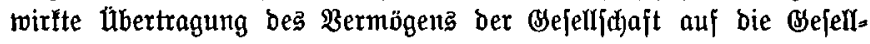
\{hafter ober auf einen bon ihnen, ber zugleich Treuhänber ber übrigen

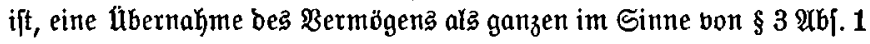
$\mathfrak{N r} 8$ Aujwos.? . . . . . . . . . . . . . . .

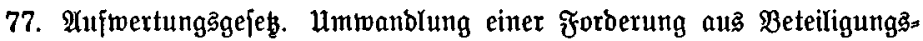
berhältnis in eine Darlehenafforberung; Redtafolgen. Berhältniz ber $\mathfrak{A b j . ~} 2$ u. 3 bes $\$ 66$ Uufwes. zueinanber ...........

78. unmöglichleit ber Leiftung; Serauggabe bes Erjabes. Luber ben $\mathfrak{A n}=$

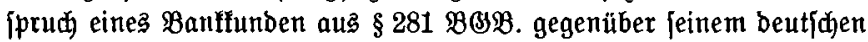
Banfier, ber bollbezahylte Effeften bes ßunben bei einem englifduen Banfier ins Depot gelegt hatte, wenn leghterer bie Effelten im Rriege zur Dechung feiner forberung gegent ben beutjijen Banfier zmangşweije berfauft hat, ohne daß biejer jemals über ben Berfaufäerlös berfügen fonnte .................. 34

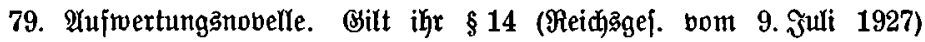

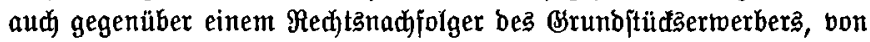
bem ober für beffen Redjunt bie Şypothef abgelöft worben ift?

80. Semmung ber Berjährung. Wann enbet bie Seemmung bei $\mathfrak{A} u f_{=}$

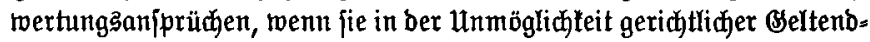
madjung folder 2 Infprüd)e iłren (srund hat? über ben Beginn bes

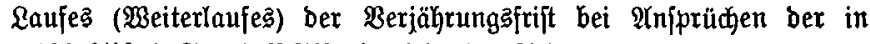

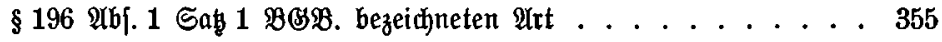


gir.

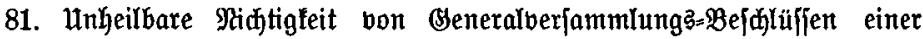

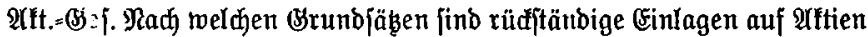
umzutwerten, wenn bie Befellfdjaft in Sonfurs geraten ift und bie

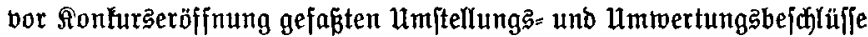
zoar im sanbelshregifter eingetragen, aber unheilbar niđtig find?

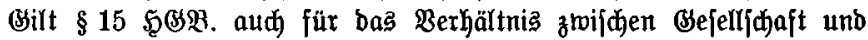
Aftionären?

83. Şödftbetragahypothef. F̧t bie Durdh fie gefidjerte Forberung aud bann eine burd Sypothef geficherte Forberung in Sinne bon $\$ 9$ AYufw (S., wenn nod nidyt jejtfteht, weldhe beftimmten forberungen

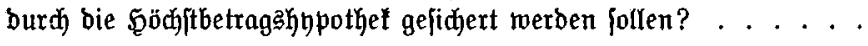

84. Dauter und Berluft bes arusftattungabefikes. Sind gegenüber ber

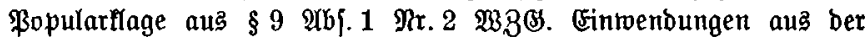
\$erjon bes ßopularflägers zuläfig, insbejonbere bie Einrebe ber Arglift? . . . . . . . . . . . . . . . . 402

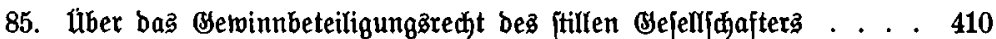

\section{b. Ianderadit.}

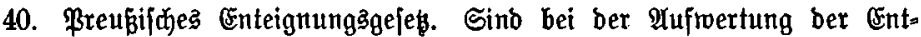

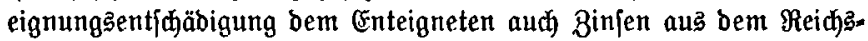
marfbetrag feit bem für bie Bertermittlung mápgebenben Stidtag zйzцipred)en? ..................

\section{S̈ffentlines Medt.}

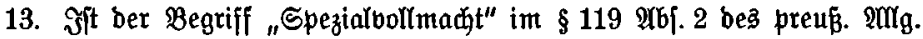
Berggelebes im Cinne einer Bollmartyt zu verftehen? luber bie Ber. fteuerung biefer "Epezialbollmad)t" ............

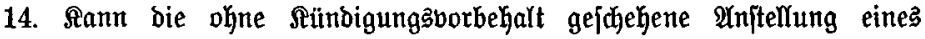

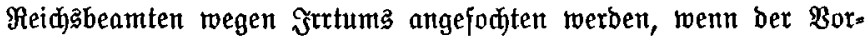
behalt nur infolge eines Berjehens nidgt in bie Anftellungäurfunte aufgenommen worben ift? . . . . . . . . . .

15. Was muß ein Beamter zu feiner Entraftung besweijen, wentt er für bas abhanbenfommen einer in feine amtlidje Obhut gelangten Sadhe ber antroortlid gemadt toiro? . . . . . . . . . . 
Ingalt.

Mr.

22. Uber Tarifbetednung bei (Shemilalien, wenn ztwar ber berjanbte Stoff eine anbere demifde Berbinbung ift als ber im Tarif genannte, wenn abcr beibe aus bem nämliden פ̂bfallerzeugniz hergeftellt unb in gleider $23 e i j e$ wirtjdaftlid berwertet werben . . . . . . . . . 101

36. Utber bie Gaftung einer Semeinbe gegenüber einer Stanfenlaffe, bie

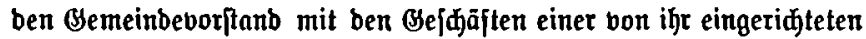
Bablitelle betraut hat, wenn ein bon jenem in ber Bablftelle be.

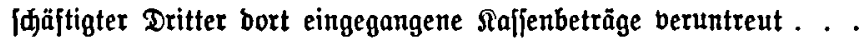

47. Ułber bie Ctaatsangehörigleit ber Frau eines burd) ben Ber[ailler

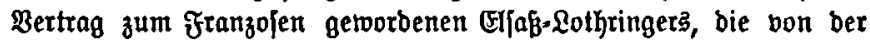
Möglidfteit franzöfin zu werben, feinen Bebraud gemadjt hat . .

51. Unter welden Borauşegungen ift bie \$olizei befugt, Pribatwohnungen zur Unterbringung von Obbaflofen zu bejalagnahmen?.....

60. Untlarheiten in Beförberungşarifen. Internationales lubereintommen über ben Eifenbahn=\{radtberfehr. Begriff ber $\mathfrak{u m}$ itationierung von Brivatwagen. . . . . . . . . . . . . . . .

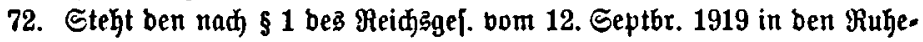
jtanb verjekten Beamten ein lebenslänglider Ilnfprud auf zebn-

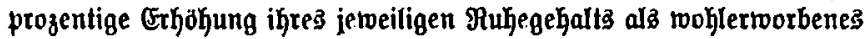

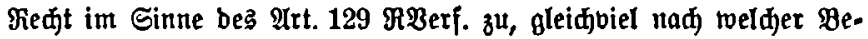
folbungsorbnung bas Ptuhegehalt berednet ift? . . . . . .

76. Berliert ein preut. Beamter burd) Berurteilung zu Feftungahaft bon längerer als einjähriger Dauer wegen Beifilfe zum ફodjucrrat aud bamn ohne weiteres fein $\mathfrak{A m t}$, wenn in Strafurteil nidht auf $\mathfrak{B e r l u j t}$ ber betleibeten offentliden Ifmter erfannt worben ift? ......

82. Miniftertuhegehalt. Einb bie 30rfdriften ber 9rt. 12 u. 13 bes württemberg. Brefełes über bie Dienftberhältniffe ber Minif̣ter, forcit

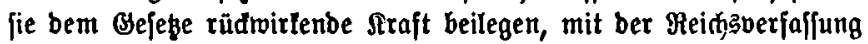
vereinbar? ................. 374

\section{G̈ertutlide Berfahren.}

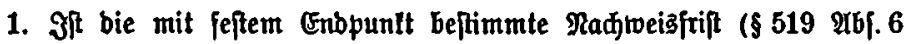
3PD.) ebenfo roie bie nad einem Beitraum beftimmte frift zu be.

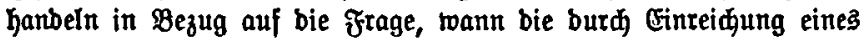

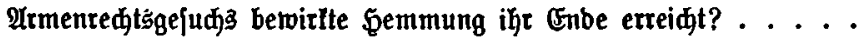

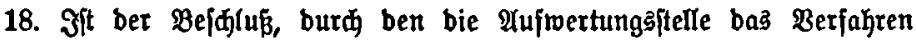
ausjeßst, mit ber einfaden ober mit ber jofortigen Bejajwetbe an-

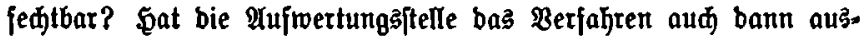


Rr.

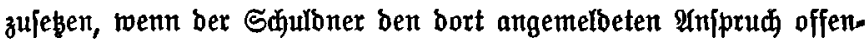
fichtlidy unbegraindeterweife beftreitet? ............

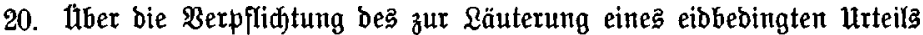
berufenen Beridits zul erneuter felbjtänbiger ßrüfung ber Gadje, wenn ber Sdjmurpflidtige von mehreren burdh ihn zu bejdwörenben Tatfad)en nadträglid eine einzelne zugefteht, im übrigen aber zux (Eibesleiftung bereit ift. Benügt in foldhem Ffalle zum भußfpruch ber für bie völlige Fibezberweigerung vorgefehenen forlge fidon ber Bweifel, ob bas frühere Beridjt biefe Sadjlage ber bölligen Eibes: berweigerung gleidggeftellt hätte? ............

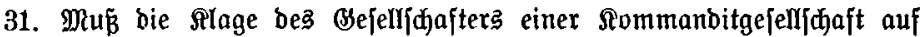

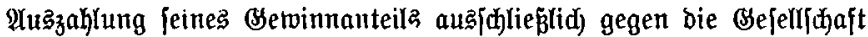
gerichtet werben, ober tommen babei aud bie anderen Beferlfdafter als Mithaftende in Betraht? . . . . . . . . . . . .

32. Shat ein vor (Finlegung ber Berufung eingereidytes armenred) frifthemmende $23 i r h u n g$, wenn ber Borjibende Desె sBerufungsggeridits

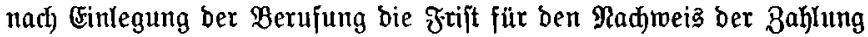

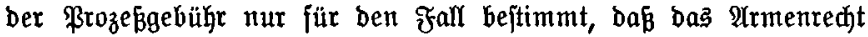
nidjt bewilligt wirb, unt wemt bie Befdäaftaftelle erft nad Berfagung

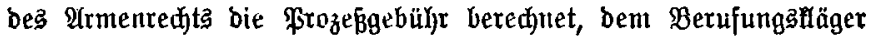
befanntgibt und von ihm einjorbert? . . . . . . . . .

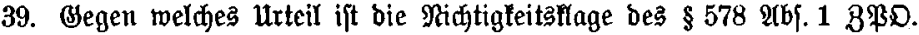
zu riđten? Steht ein bie Revijion als unzuläffig verwerfender $\mathfrak{B} e=$ ¡d

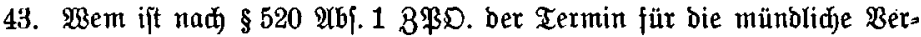
hanblung befannt zu machen? Begriff ber \$nitanz in $\$ \$ 176$ und 210a 3ßO. Unabrenbbarer 3 ufall in $\$ 233$ 965. 1 baf. . . . .

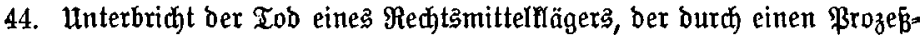
bebollmädtigten vertreten ift, ben lauf ber Frift zum Nad)tweis ber Bahlung ber zu entrichtenben Prozeggebüht?.........

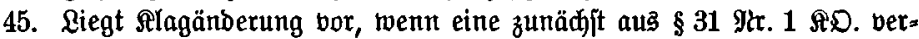

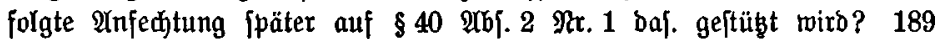

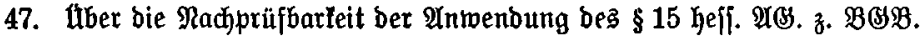
in der Rebifionsinftarz . . . . . . . . . . . . . . . 198

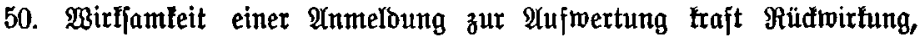

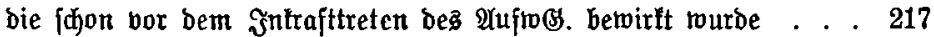

55. Buftellung nicht verfünbeter Urteile. Burüđnahme eines Red)tamittels unter $\mathscr{A n g a b e}$ Des Brumbez. Wiebereinjebung in ben borigen Stand

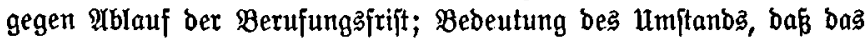

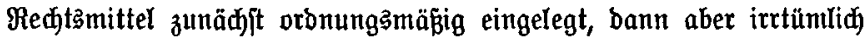

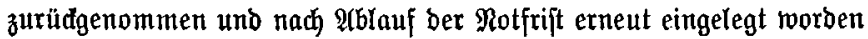


Rt.

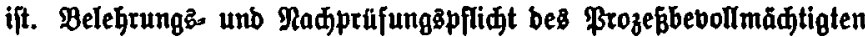

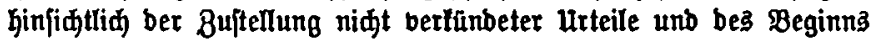
ber Bechtsmittelfrift . . . . . . . . . . . . . 243

61. Ift im Berfahren bor ber $\mathfrak{A}$ ufwertungajtelle nad $\mathfrak{A b l a u f}$ ber frift für bie jofortige Bejdwerbe nod eine Bejdwerbe als Ânjdiukbefdrverbe zuläffig? . . . . . . . . . . . . . 274

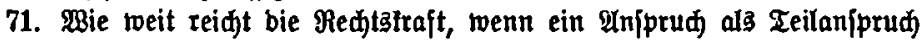
erboben unb teilweife zugefprođen, teilweife abgerwiejen wirb? . . 317

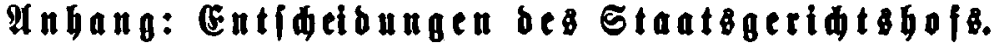

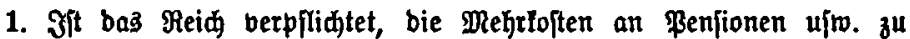
tragen, bie burd Erftredung ber fädj]. Berorbnung vom 15. Oltober 1926 auf bie gutpenfionãre ber auf bas Reid tabergegangenen fädj]. Boll- unb Steuerberwaltung entfteken? ............ 1*

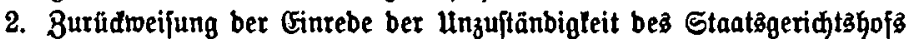

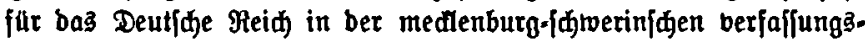
redtlidgen Streitfađje betreffenb bie Berfaffungabibrigleit bes Ranb.

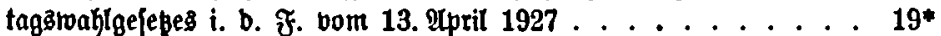

Sa⿱⺌兀regifter. . . . . . . . . . . . . . . . . 413

Gejęegregifer. . . . . . . . . . . . . . . . . . 422

8ujammenftellung nad ber Beitfolge .......... 434

8 ujammenftellung na山 Dberlanbegigeridtsbegirfen . . . . . . . . 439

Beribtigungett. . . . . . . . . . . . . . . . . . . . 440 